\title{
CD44 variant isoforms are specifically expressed on peripheral blood lymphocytes from asthmatic patients
}

\author{
CHUN YANG $^{1^{*}}$, HONGYAN LIANG ${ }^{1,2^{*}}$, HONGLI ZHAO ${ }^{3}$ and XIAOFENG JIANG ${ }^{1,2}$ \\ ${ }^{1}$ Department of Clinical Biochemistry Laboratory, The 4th Affiliated Hospital of Harbin Medical University; \\ ${ }^{2}$ Heilongjiang Province Key Laboratory of Molecular Imaging, Harbin; \\ ${ }^{3}$ The Department of Internal Medicine, Harbin Children's Hospital, Harbin, P.R. China
}

Received January 11, 2012; Accepted March 22, 2012

DOI: $10.3892 /$ etm. 2012.543

\begin{abstract}
Asthma is a disease characterized by chronic airway inflammation, and Th2 cells play a critical role in initiating and sustaining asthmatic inflammation. It has been shown that CD44 expressed on $\mathrm{CD}^{+}{ }^{+} \mathrm{T}$ cells plays a critical role in the accumulation of antigen-specific Th2 cells in the development of airway hyperresponsiveness induced by antigen challenge in the airways. The aim of this study was to determine whether there are specific CD44 variant isoforms (CD44v) expressed on lymphocytes from asthmatic patients. We collected whole blood samples from 103 normal subjects, 165 subjects with asthma and 104 with pneumonia. Peripheral blood lymphocyte isolation was performed, and total RNA was extracted from the isolated lymphocytes, using nested PCR for specific CD44v amplification on lymphocytes. Demographic variables were analyzed using linear regression in order to determine whether the expression of CD44v was correlated with these demographic features. The nested PCR results revealed that CD44v5 was expressed by $55.2 \%$ of asthma patients, which was significantly higher than levels of expression in the other groups. Lower percentages of individuals in the normal subject group exhibited expression of CD44v5 and CD44v6. The data demonstrated that the percentage of individuals in the pneumonia group expressing CD44v5 was $29.0 \%$, but a higher percentage of these patients expressed CD44v6. CD44v5 expression was positively correlated with $\operatorname{IgE}$ levels $(\mathrm{p}=0.032)$ in the asthmatic patient group, and CD44v6 was significantly positively correlated with the neutrophil count $(\mathrm{p}<0.05)$. CD44v5 was expressed by a higher proportion of asthmatic patients than other subjects and thus may play an important role in the pathogenesis of asthma.
\end{abstract}

Correspondence to: Dr Xiaofeng Jiang, Department of Clinical Biochemistry Laboratory, The 4th Affiliated Hospital of Harbin Medical University, 37 Yiyuan Street, Nangang District, Harbin 150001, P.R. China

E-mail: jiangxiaofeng12359@yahoo.com.cn

${ }^{*}$ Contributed equally

Key words: asthma, peripheral blood lymphocytes, CD44v5, CD44v6
These findings may offer a new target for the diagnosis and treatment of asthma and may also provide insights into the mechanisms of asthma development.

\section{Introduction}

Asthma is a chronic airway inflammatory disease characterized by inflammation, airway hyperresponsiveness (AHR), reversible airway obstruction and elevated production of cytokines (1). AHR is mainly caused by lung inflammatory responses in asthma. Eosinophil (Eos) and T-lymphocyte $\left(\mathrm{CD}^{+}\right)$infiltration and the increased secretion of inflammatory mediators during asthma adversely affect the large and small airways (2). In particular, Th2 cells play a critical role in initiating and sustaining asthmatic inflammation (3). Increased numbers of activated $\mathrm{T}$ cells are found in the peripheral blood of patients during acute episodes of asthma (4).

CD44-hyaluronate interactions are able to promote extravasation and egress of antigen-activated lymphocytes in inflamed vascular beds (5). Furthermore, Katoh et al demonstrated that CD44 plays an important role in the accumulation of $\mathrm{T}$ helper type 2 (Th2) cells in the airways of mice (6). CD44 expressed on $\mathrm{CD}^{+} \mathrm{T}$ cells plays a critical role in the accumulation of antigen-specific Th2 cells in the development of AHR induced by antigen challenge in the airways (7).

Adhesion molecule CD44 is a type 1 cell surface transmembrane glycoprotein which is encoded on the short arm of chromosome 11 (8). The CD44 family contains two types of CD44: a standard form (CD44s) and variant isoforms (CD44v).

The genetic sequence is composed of two groups of exons. One group comprising exons 1-5 and 16-20 is expressed together on all cell types as the standard form. The non-variant exon mRNA encoded isoform has been termed CD44s. The 9 variable exons v2-v10 (exons 7-15, vl was isolated in rats and is not present in humans) may be alternatively spliced and included within the standard exons at an insertion site between exons 5 and 16, which code for a variety of proteins by selecting certain exons within the sequence - for example, CD44v can contain one or more variant regions, such as CD44v3 or CD44v4-7.

Although the specific functions of CD44v remain unclear in humans, CD44 and its many variant isoforms were demonstrated to exert some of their functions through docking OPN 
and growth factors to their cognate cell surface receptors or substrates (9-11).

It was found that there is a significant increase in the expression of anti-apoptotic Bcl-2 protein and a decrease in the expression of pro-apoptotic protein in asthmatic patients when compared to normal individuals. Thus, inhibition of apoptosis may be one of the reasons for the chronic persistent inflammation in the airways of asthmatic patients (12). This may result in the increase in memory $\mathrm{T}$ cells. It has been demonstrated that $\mathrm{CD} 45 \mathrm{RO}^{+}$may provide pro-inflammatory signals that contribute to the persistent airway inflammation of asthma (13).

The CD44v also promote ECM-derived survival signals mediated through integrin activation through OPN-CD44V interaction (14). As such, CD44v should play a vital role in the pathogenesis of asthma and there may even be some CD44v uniquely expressed on the peripheral blood lymphocyte cells of asthmatic patients. The aim of this study was to determine whether there are specific CD44 isoforms expressed on peripheral blood lymphocytes of asthmatic patients compared with normal individuals and pneumonia patients.

\section{Patients and methods}

Subjects. We collected blood samples from individuals (103 normal, 165 with asthma and 104 with pneumonia) who presented to The 4th Affiliated Hospital of Harbin Medical University from March 2010 to May 2011. The normal controls had no history of asthma, other allergic diseases or AHR. They had normal total IgE values and normal lung function tests.

We enrolled patients with asthma who were diagnosed on the basis of a history of dyspnea and wheezing during the previous 12 months and lung function tests $>12 \%$ FEV1 following $\beta 2$-agonist inhalation. Patients with pneumonia were recruited on the basis of definite clinical diagnosis by chest X-ray and/or computerized tomography, which revealed bilateral diffused pulmonary infiltrations.

A routine blood test (including eosinophil, neutrophil, monocyte and lymphocyte counts) was performed for each participant. Approval of the ethics committee of our institution was obtained for this study. Written informed consent was obtained from all participants.

Lymphocyte isolation. Whole blood samples $(10 \mathrm{ml})$ were drawn from the antecubital vein for lymphocyte isolation, and mononuclear cells were isolated by gradient centrifugation on Histopaque-1083 (Sigma, St. Louis, MO, USA) according to the manufacturer's instructions. The unadhered lymphocytes were harvested. Centrifugation was performed at $600 \mathrm{x} \mathrm{g}$ for $15 \mathrm{~min}$ at room temperature. Following centrifugation, the cells were washed twice. The isolated lymphocytes were confirmed by flow cytometry with fluorescently labeled antibodies and stained with CD3 and CD19 (BD Pharmigen, San Diego, CA, USA). The lymphocytes used in our experiments had a purity of $>90 \%$.

Reverse transcriptase and nested PCR for specific CD $44 \mathrm{v}$ on lymphocytes. Total RNA was extracted from the isolated lymphocytes. TRIzol reagent was used according to the manufacturer's instructions (Invitrogen, Carlsbad, CA, USA) and

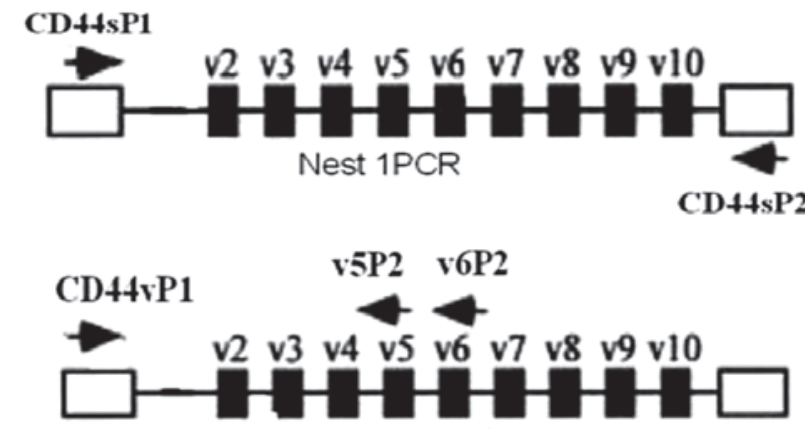

Nest 2 PCR

Figure 1. An illustration of the protocol for nested PCR of the CD44v.

then RNA was reverse transcribed into cDNA using M-MLV Reverse Transcriptase (Promega Corporation, Madison, WI, USA).

Nest 1. Plasmodium genus-specific PCR amplification was performed in $25 \mu \mathrm{l}$ reaction mixtures, $0.25 \mu \mathrm{M}$ of each primer (primer for CD44s), 1.25 unit Taq DNA polymerase (Promega), $2 \mu \mathrm{l}$ of cDNA template, and the final volume was adjusted to $25 \mu \mathrm{l}$ with deionized water. The cycling conditions of nest 1 PCR amplification of total CD44 isoforms were as follows: initial denaturation at $95^{\circ} \mathrm{C}$ for $3 \mathrm{~min}, 10$ cycles of denaturation at $95^{\circ} \mathrm{C}$ for $30 \mathrm{sec}$, annealing at $59^{\circ} \mathrm{C}$ for $30 \mathrm{sec}$, and extension at $72^{\circ} \mathrm{C}$ for $40 \mathrm{sec}$, followed by final extension at $72^{\circ} \mathrm{C}$ for $7 \mathrm{~min}$.

Nest 2. Two microliters of the nest 1 diluted amplification product (diluted to 50 times with deionized water) was used as the template DNA in the nest 2 PCR amplification. The concentration of the constituents and nest 2 primers were similar to nest 1 for the annealing temperature which was $57^{\circ} \mathrm{C}$ for $\mathrm{CD} 44 \mathrm{v}$ specific primers (CD44v5 and CD44v6) and PCR amplification for specific primers consisted of 30 cycles (Fig. 1). The sequences of the specific primers are presented in Table I.

The nest 2 PCR amplification products were analyzed by gel electrophoresis on a $2 \%$ agarose gel and visualized by staining with ethidium bromide $(4 \mu \mathrm{g} / \mathrm{ml})$ and ultraviolet transillumination.

The levels of CD44s and GAPDH mRNA were measured by quantitative RT-PCR using the SYBR-Green PCR Core reagents kit (Applied Biosystems, USA) and specific primers on a DA7600 PCR amplifier (DAAN, China). The CD44 (CD44s, CD44v5 and CD44v6) primers were designed according to the reported gene sequences of Homo sapiens CD44 molecule, transcript variant 1, mRNA (NM_000610.3) and GAPDH (NM_002046.3). The sequences of the specific primers are presented in Table I.

The PCR reactions ( $50 \mathrm{ml} /$ tube, in duplicate) were denatured at $95^{\circ} \mathrm{C}$ for $2 \mathrm{~min}$ and subjected to 30 cycles of $95^{\circ} \mathrm{C}$ for $30 \mathrm{sec}, 57^{\circ} \mathrm{C}$ for $30 \mathrm{sec}$ and $72^{\circ} \mathrm{C}$ for $30 \mathrm{sec}$. The relative levels of mRNA transcripts were analyzed by normalizing the values of individual samples to GAPDH.

Data analysis. The clinical parameters for IgE were converted into log-based values in order to produce a normal distribution for the statistical analyses, and then the data were 
Table I. Sequences of the specific primers.

\begin{tabular}{lc}
\hline Gene & Primer sequence \\
\hline CD44s (Nest1) & \\
P1 5'-3' & AAGACATCTACCCCAGCAACCC \\
P2 5'-3' & TGCAGTAACTCCAAAGGACCCA \\
CD44'5 (Nest2) & CTGAAGACATCTACCCCAGCAAC \\
P1 5'-3' & ATAAGCAGTGGTGCCATTTCTG \\
P2 5'-3' & \\
CD44v6 (Nest2) & \\
P1 5'-3' & CTGAAGACATCTACCCCAGCAAC \\
P2 5'-3' & TTGCCAAACCACTGTTCCTTC \\
GAPDH (qPCR) & \\
P1 5'-3' & AAGGTCGGAGTCAACGGATTTGG \\
P2 5'-3' & TTGGAGGGATCTCGCTCCTGGAA \\
\hline
\end{tabular}

Table II. Clinical characteristics of the study participants.

\begin{tabular}{lccr}
\hline Clinical parameters & Normal & Asthmatic & Pneumonia \\
\hline Gender $(\mathrm{M} / \mathrm{F})$ & $59 / 54$ & $71 / 94$ & $56 / 48$ \\
Age $($ years $)$ & $45.4 \pm 13.5$ & $43.4 \pm 12.1$ & $51.7 \pm 10.6$ \\
Eos $\left(\mathrm{x} 10^{9}\right)$ & $0.31 \pm 0.22$ & $5.21 \pm 2.15^{\mathrm{a}}$ & $0.24 \pm 0.17$ \\
Mono $\left(\mathrm{x} 10^{9}\right)$ & $0.28 \pm 0.03$ & $0.31 \pm 0.03$ & $0.56 \pm 0.04$ \\
$\operatorname{Lym}\left(\mathrm{x} 10^{9}\right)$ & $2.4 \pm 0.33$ & $3.1 \pm 0.54^{\mathrm{b}}$ & $3.5 \pm 0.61^{\mathrm{b}}$ \\
Neu $\left(\mathrm{x} 10^{9}\right)$ & $3.6 \pm 1.42$ & $3.4 \pm 2.11$ & $9.6 \pm 2.33^{\mathrm{a}}$ \\
$\log \operatorname{IgE}\left(\mathrm{IU} / \mathrm{ml}^{-1}\right)$ & $0.7 \pm 0.09$ & $2.1 \pm 0.32^{\mathrm{a}}$ & $0.65 \pm 0.11$ \\
\hline
\end{tabular}

M, male; F, female. Eos, eosinophil count; mono, monocyte count; lym, lymphocytes count; neu, neutrophil count; IgE, immunoglobulin E. Both the asthma and pneumonia groups were compared with the normal group, according to the Mann-Whitney U-test. ${ }^{a} \mathrm{P}-\mathrm{value}<0.001$; ${ }^{\mathrm{b}} \mathrm{P}$-value $<0.05$. Values are expressed as the mean $\pm \mathrm{SD}$.

analyzed using the Mann-Whitney U-test. The $\chi^{2}$ test was used to examine the difference in clinical indices among the three groups. The demographic variables of the subjects (for example, gender and age) were analyzed by linear regression in order to determine whether or not the expression of CD44v was correlated with the clinical features. Values are expressed as the means $\pm \mathrm{SD}$. The expression of CD44v among the three groups was statistically compared using $\chi^{2}$ test and Student-Newman-Keuls. Data were analyzed using the SAS 9.1 statistical program. A p-value $<0.05$ was considered to indicate statistical significance.

\section{Results}

We divided the subjects into three groups: 113 normal subjects, 165 subjects with asthma and 104 subjects with pneumonia. We compared the gender, age and blood indices among the three groups, finding no differences in gender and age. The blood examination results were different in the three groups. The Eos and the IgE levels were significantly higher in the asthmatic patients than the levels in the other groups $(\mathrm{p}<0.001$, compared with the normal and pneumonia groups). In the normal group, the lymphocyte count was slightly lower than the count in the other groups $(\mathrm{p}<0.05)$, and the neutrophil count in the pneumonia group was obviously higher than that in the other groups $(\mathrm{p}<0.001)$ (Table II).

From the results of the nested PCR of the peripheral lymphocyte cells of the subjects, we demonstrated that expression levels of CD44s, CD44v5, and CD44v6 differed among the normal, asthmatic and pneumonia groups. The level of CD44s expression was not significantly difference among the normal, asthmatic and pneumonia groups as determined by quantitative real-time PCR (Fig. 2). However, expression of CD44v was clearly different (Table III). CD44v5 was expressed in $55.2 \%(91 / 165)$ of asthma patients which was significantly higher than the other groups. The expression level of CD44s was identical in the three groups. CD44v5 expression was found to be independent of the levels of CD44s. We also investigated the expression of other CD44v (data not shown), but the results did not reveal any significant difference among the groups.

In contrast, in the normal group only $21.2 \%(24 / 113)$ of individuals expressed CD44v5, and a low percentage of $23.0 \%$ (26/113) also expressed CD44v6. The data revealed that $26.0 \%$ 


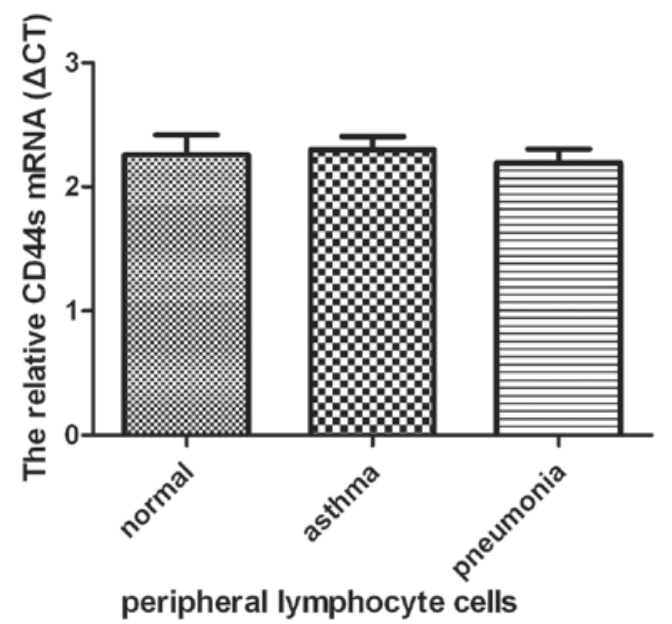

Figure 2. The relative levels of CD44 mRNA of peripheral blood lymphocytes cell were determined by quantitative RT-PCR.

Table III. Expression of CD44 isoforms on peripheral blood lymphocyte cells in normal, asthmatic and pneumonia patients.

\begin{tabular}{lccc}
\hline & $\mathrm{n}$ & $\mathrm{CD} 44 \mathrm{v} 5^{+}$ & $\mathrm{CD} 44 \mathrm{v} 6^{+}$ \\
\hline Normal & 113 & 24 & 26 \\
Asthmatic & 165 & 91 & 23 \\
Pneumonia & 104 & 27 & 67 \\
\hline
\end{tabular}

(27/104) of the pneumonia group weakly expressed CD44v5, while a high proportion of $64.4 \%$ (67/104) patients expressed CD44v6. The high expression of CD44v6 appears to be independent of CD44s.

The $\chi^{2}$ tests demonstrated that $\mathrm{CD} 44 \mathrm{v}$ was differentially expressed among the three groups $\left(\chi^{2}=117.710, p<0.0001\right)$. Student-Newman-Keuls tests were performed, which indicated that the asthmatic patients had a significantly higher CD44v5 expression than the other groups $(\mathrm{p}<0.001)$, and CD44v6 expression was highly expressed in the subjects with pneumonia $(\mathrm{p}<0.001)$.

The data were analyzed by multiple logistic regression, and correlations between age and gender and the expression of CD44s, CD44v5, and CD44v6 were not found. Regression analysis revealed that CD44v5 expression was not significantly correlated with blood indices such as Eos, neutrophil and lymphocyte counts, while a positive correlation with $\operatorname{IgE}$ level $(\mathrm{p}=0.032)$ was noted in the asthma group, indicating that the expression of CD44v5 is correlated with the extent of the allergic state. CD44v6 was significantly positively correlated with the neutrophil count $(p<0.05)$. The reason for this observation is unclear, but it may be explained by the CD44v6 expression of $\mathrm{T}$ cells inducing Th1 inflammation in pneumonia.

\section{Discussion}

According to our data, CD44v5 was found to be expressed on the T cells of asthmatic patients and CD44v6 was expressed in patients with pneumonia. The expression of CD44v5 was positively correlated with the $\operatorname{IgE}$ level. These data have not previously been reported. Although the reason for these observations are still unknown, it may be suggested that CD44v5 expression correlates with the extent of the allergic state. CD44v5 expressed on $\mathrm{T}$ cells may participate in Th2 polarization or may enhance Th2 cytokine secretion. Certainly there is a need to confirm our findings with further research.

There are three possible explanations for the differential expression of CD44 in peripheral lymphocyte cells between asthmatic and pneumonia patients. First, they may play varying roles in the $\mathrm{T}$ cell activation of the Th1/Th2 condition respectively. Hegde et al (15) found that mobilization of CD44 in allogeneic dendritic cell-T cell immunological synapse plays a key role in T cell activation. Upon TCR engagement, TCR and important signaling molecules such as Lck, Fyn, $\mathrm{PKC}$, phospholipase $\mathrm{C}$ and linker for activation of $\mathrm{T}$ cells were found to be recruited to the raft aggregates at the T cell-APC contact $(16,17)$. CD44 has been shown to partition into lipid rafts (18), associated, activated tyrosine kinases p59fyn and p56lck $(19,20)$, but there should be different signal transduction pathways among the various isoforms.

The present controversy among studies may be due to their omitting the various CD44 isoforms which could play a variety of roles in the different immunoreactions.

Secondly, CD44 as an adhesion molecule should take part in the $\mathrm{T}$ cell infiltration into the lung and the Th2 memory $\mathrm{T}$ cell resident in the lung. The adhesion between inflammatory cell and bronchial smooth muscle (BSM) cell types may contribute to long-term effects leading to hyperresponsiveness and airway remodeling but the mechanism by which this occurs remains unknown. Therefore, CD44v5 may be the specific isoform which interacts with the other adhesion molecules on BSM lung tissue to finally induce $\mathrm{T}$ cell infiltration.

Thirdly, it is well known that subpopulations of allergenspecific memory Th2 cells are generated during an immune response to allergens. Following recovery from allergic asthma, allergen-specific memory Th2 cells reside within the lungs of mice for their remaining life span rather than going into programmed cell death. Upon specific allergen exposure, these allergen-specific Th2-skewed memory $\mathrm{T}$ cells rapidly induce the Th2 inflammation (including eosinophilic mucus production, airway hyperresponsiveness and allergen-specific IgE production) (21). The Th2 cells do not undergo apoptosis following immunoreaction, which is the main cause for the elevation of Th2-skewed memory T cells. Fas is a major trigger for apoptosis, especially in activated immune cells. There is evidence that the CD44v region may be involved in apoptosis (22).

Mielgo et al (23) demonstrated that CD44v6 and v9 colocalize and interact with Fas in the presence or absence of FasL. Based on these findings, we propose a model in which CD44v interacts extracellularly with Fas, preventing FasL binding and consequently Fas death signaling in the Jurkat cell line. The CD44v5 expressed on the peripheral lymphocyte cells of asthma patients may be an anti-apoptosis molecule; by CD44v5 interacting extracellularly with Fas, this reduced the Th2 cell skewed into memory $\mathrm{T}$ cells.

In this study, we demonstrated that $64.4 \%$ of the pneumonia patients expressed CD44v6. Previous studies demonstrated 
that CD44v7 is expressed on $\mathrm{T}$ cells and macrophages in T-helper-1 (Th1)-mediated chronic inflammation and autoimmune diseases (24). CD44v may also have a ligation with OPN that induces an inside-out signaling transduced through Src, leading to integrin activation, which in turn facilitates cell adhesion and enhances matrix survival signaling (25).

In the present study, the normal subjects also expressed CD44v5 and CD44v6. This may be due to individual differences and the normal subjects we enrolled may not have been in perfect health. Equally we could not guarantee that the asthma and pneumonia patients did not have co-morbidities. Following this study, we will proceed to verify our findings in an animal model and investigate the mechanism of CD44v in asthma.

CD44v5 was expressed by a higher proportion of asthmatic patients than the other subjects and should also play an important role in the pathogenesis of asthma, which may provide a new target for diagnosis of asthma and shed light on the mechanism of asthma. This finding opens new strategies for therapeutic intervention in asthma.

\section{Acknowledgements}

This study was supported by the National Natural Science Foundation of China (No. 81171657).

\section{References}

1. Bochner BS, Undem BJ and Lichtenstein LM: Immunological aspects of allergic asthma. Annu Rev Immunol 12: 295, 1994.

2. Fabbri LM, Romagnoli M, Corbetta L, et al: Differences in airway inflammation in patients with fixed airflow obstruction due to asthma or chronic obstructive pulmonary disease. Am J Respir Crit Care Med 167: 418-424, 2003.

3. Robinson DS, Hamid Q, Ying S, Tsicopoulos A, Barkans J, et al: Predominant TH2-like bronchoalveolar T-lymphocyte population in atopic asthma. N Engl J Med 326: 298, 1992.

4. Shalaby KH and Martin JG: Overview of asthma: the place of the T cell. Curr Opin Pharmacol 10: 218-225, 2010.

5. DeGrendele HC, Estess P and Siegelman MH: Requirement for CD44 in activated T cell extravasation into an inflammatory site. Science 278: 672-675, 1997.

6. Katoh S, Ishii N, Nobumoto A, et al: Galectin-9 inhibits CD44hyaluronan interaction and suppresses a murine model of allergic asthma. Am J Respir Crit Care Med 176: 27-35, 2007.

7. Katoh S, Kaminuma O, Hiroi T, et al: CD44 is critical for airway accumulation of antigen-specific Th2, but not Th1, cells induced by antigen challenge in mice. Eur J Immunol 41: 3198-3207, 2011

8. Goodfellow PN, Banting G, Wiles MV, et al: The gene, MIC4, which controls expression of the antigen defined bymonoclonal antibody F10.44.2 is on human chromosome 11. Eur J Immunol 12: 659-663, 1982.
9. Ponta H, Sherman L and Herrlich PA: CD44: from adhesion molecules to signalling regulators. Nat Rev Mol Cell Biol 4: 33-45, 2003.

10. Denhardt DT, Noda M, O'Regan AW, et al: Osteopontin as a means to cope with environmental insults: regulation of inflammation, tissue remodeling, and cell survival. J Clin Invest 107: 1055-1061, 2001.

11. Bennett KL, Jackson DG, Simon JC, et al: CD44 isoforms containing exon $\mathrm{v} 3$ are responsible for the presentation of heparin binding growth factor. J Cell Biol 128: 687-698, 1995.

12. Xue J, Xu Y and Zhang Z: Lymphocyte apoptosis in asthmatic patients and its molecular mechanism. Zhonghua Jie $\mathrm{He} \mathrm{He} \mathrm{Hu}$ Xi Za Zhi 22: 555-557, 1999 (In Chinese).

13. Lamb JP, James A, Carroll N, et al: Reduced apoptosis of memory T-cells in the inner airway wall of mild and severe asthma. Eur Respir J 26: 265-270, 2005.

14. Lee JL, Wang MJ, Sudhir PR, et al: Osteopontin promotes integrin activation through outside-in and inside-out mechanisms: OPN-CD44V interaction enhances survival in gastrointestinal cancer cells 67: 2089-2097, 2007.

15. Hegde VL, Singh NP, Prakash S, et al: CD44 mobilization in allogeneic dendritic cell-T cell immunological synapse plays a key role in T cell activation. J Leukoc Biol 84: 134-142, 2008.

16. Xavier R, Brennan T, Li Q, et al: Membrane compartmentation is required for efficient T cell activation. Immunity 8: 723-732, 1998.

17. Viola A, Schroeder S, Sakakibara Y, et al: T lymphocyte costimulation mediated by reorganization of membrane microdomains. Science 283: 680-682, 1999.

18. Neame SJ, Uff CR, Sheikh H, et al: CD44 exhibits a cell type dependent interaction with Triton X-100 insoluble, lipid rich, plasma membrane domains. J Cell Sci 108: 3127-3135, 1995.

19. Ilangumaran S, Briol A and Hoessli DC: CD44 selectively associates with active Src family protein tyrosine kinases Lck and Fyn in glycosphingolipid-rich plasma membrane domains of human peripheral blood lymphocytes. Blood 91: 3901-3908, 1998.

20. Foger N, Marhaba R and Zoller M: Involvement of CD44 in cytoskeleton rearrangement and raft reorganization in T cells. J Cell Sci 114: 1169-1178, 2001.

21. Mojtabavi N, Dekan G, Stingl G and Epstein MM: Long-lived Th2 memory in experimental allergic asthma. J Immunol 169: 4788-4796, 2002.

22. Günthert U and Johansson B: CD44 - a protein family involved in autoimmune diseases and apoptosis. Immunologist 8: 106-109, 2001.

23. Mielgo A, van Driel M, Bloem A, et al: A novel antiapoptotic mechanism based on interference of Fas signaling by CD44 variant isoforms. Cell Death Differ 13, 465-477, 2006.

24. Wittig BM, Johansson B, Zoller M, et al: Abrogation of experimental colitis correlates with increased apoptosis in mice deficient for CD44v7. J Exp Med 191: 2053-2063, 2000.

25. Hoffmann U, Heilmann K, Hayford C, et al: CD44v7 ligation downregulates the inflammatory immune response in Crohn's disease patients by apoptosis induction in mononuclear cells from the lamina propria. Cell Death Differ 14, 1542-1551, 2007. 